\title{
Quantum Information and General Relativity
}

\author{
Asher Peres \\ Technion - Israel Institute of Technology, Haifa
}

\begin{abstract}
The Einstein-Podolsky-Rosen paradox (1935) is reexamined in the light of Shannon's information theory (1948). The EPR argument did not take into account that the observers' information was localized, like any other physical object. General relativity introduces new problems: there are horizons which act as on-way membranes for the propagation of quantum information, in particular black holes which act like sinks.
\end{abstract}

\section{SPQR}

It is a pleasure to dedicate this article to Francesco DeMartini on the occasion of his 70th birthday. Francesco is a magician of experimental demonstrations for Spin Polarized Quantum Radiation. I am only a theorist, which is much easier.

When I was a young man, my thesis adviser was Nathan Rosen, and the subject was the existence of gravitational radiation in general relativity. Only much later, I seriously learnt quantum mechanics, and still much later information theory. I now want to return to my roots and try to combine all these subjects together.

\section{The Einstein-Podolsky-Rosen dilemma}

Rosen had been a post-doc of Einstein at the Institute for Advanced Studies in Princeton. One day, at the traditional 3 o'clock tea, as Rosen mentioned to Einstein a fundamental issue of interpretation related to entangled wave-functions, Einstein immediately saw the implications for his long standing disagreement with Bohr. As they discussed the problem, Boris Podolsky joined the conversation, and later proposed to write an article. Einstein acquiesced. When he later saw the text, he disliked the formal approach, but agreed to its publication [1]. Then, as soon as the EPR article appeared, Podolsky relased its contents to the New York Times (4 May 1935, page 11) in a way implying that the authors had found that quantum mechanics was faulty. This infuriated Einstein, who after that no longer spoke with Podolsky.

The EPR "paradox" drew immediate attention. Niels Bohr [2] found the reasoning faulty, because it contradicted his complementarity principle. Bell, in his first article on hidden variables and contextuality [3, wrote "the Einstein-Podolsky-Rosen paradox is resolved in the way which Einstein would have liked least." Actually, the example given by Bell in the proof of his celebrated theorem [4] is based on a much simpler entangled system: two spin- $\frac{1}{2}$ particles in a singlet state [5]. 
The EPR article was not wrong, but it had been written too early. Only some years later, in 1948, Claude Shannon published his theory of information [6] (and it took many more years before the latter was included in the physicist's toolbox). Shannon was employed by the Bell Telephone Company and his problem was to make communication more efficient. Shannon showed that information could be given a quantitative measure, that he called entropy. It was later proved that Shannon's entropy is fully equivalent to ordinary thermodynamical entropy [7]. Information can be converted to heat and can perform work. Information is not just an abstract notion [8]. It requires a physical carrier, and the latter is (approximately) localized. After all, it was the business of the Bell Telephone Company to transport information from one telephone to another telephone, in a different location.

In the EPR article, the authors complain that "it is possible to assign two different wave functions to ... the second system," and then, in the penultimate paragraph, they use the word simultaneous no less than four times, a surprising expression for people who knew very well that this term was undefined in the theory of relativity. Let us examine this issue with Bohm's singlet model. One observer, conventionally called Alice, measures the $z$-component of the spin of her particle and finds $+\hbar / 2$. Then she immediately knows that if another distant observer, Bob, measures (or has measured, or will measure) the $z$-component of the spin of his particle, the result is certainly $-\hbar / 2$. One can then ask: when does Bob's particle acquire the state with $s_{z}=-\hbar / 2$ ?

This question has two answers. The first answer is that the question is meaningless this is undoubtedly true. The second answer is that, although the question is meaningless, it has a definite answer: Bob's particle acquires this state instantaneously. This then raises a new question: in which Lorentz frame is it instantaneous? Here, there is also a definite answer: it is instantaneous in the Lorentz frame that we arbitrarily choose to perform our calculations 9]. Lorentz frames are not material objects: they exist only in our imagination.

When Alice measures her spin, the information she gets is localized at her position, and will remain so until she decides to broadcast it. Absolutely nothing happens at Bob's location. From Bob's point of view, all spin directions are equally probable, as can be verified experimentally by repeating the experiment many times with a large number of singlets without taking in consideration Alice's results. Thus, after each one of her measurements, Alice assigns a definite pure state to Bob's particle, while from Bob's point of view the state is completely random ( $\rho$ is proportional to the unit matrix). It is only if and when Alice informs Bob of the result she got (by mail, telephone, radio, or by means of any other material carrier, which is naturally restricted to the speed of light) that Bob realizes that his particle has a definite pure state. Until then, the two observers can legitimately ascribe different quantum states to the same system. For Bob, the state of his particle suddenly changes, not because anything happens to that particle, but because Bob receives information about a distant event. Quantum states are not physical objects: they exist only in our imagination.

\section{Curved Spacetime}

It took Einstein more than ten years of intensive work to progress from special relativity to general relativity. Despite its name, the latter is not a generalization of the special theory, but a radically different construct: spacetime is not only a passive arena where dynamical processes take place, but has itself a dynamical nature. At this time, there 
is no satisfactory quantum theory of gravitation (in spite of seventy years of efforts by leading theoretical physicists).

Concepts of quantum information were recently invoked in several problems of quantum gravity and quantum cosmology. Even if we still consider spacetime as a passive arena endowed with a Riemannian metric, instead of the Minkowski metric of special relativity, the difference between them is essential: it is necessary to introduce notions of topology, because it may be impossible to find a single coordinate system that covers all of spacetime. To achieve that result, it may be necessary to use several coordinate patches, sewed to each other at their boundaries. Then in each patch, the metric is not geodesically complete: a geodesic line stops after a finite length, although there is no singularity there. The presence of singularities (points of infinite curvature) is another consequence of Einstein's gravitational equations. It is likely that these equations, which were derived and tested for the case of moderate curvature, are no longer valid under such extreme conditions. I shall not speculate on this issue, and shall restrict my attention to the behavior of quantum systems in the presence of horizons, in particular of black holes that result from concentrations of matter so large that their gravity pull prevents the escape of light. In other words, a future horizon is formed. While Unruh's horizons [10] appear for uniformy accelerated observers whose asymptotic speed approaches $c$, a black hole horizon affects every observer.

Even in classical general relativity, there is a serious difficulty with the second law of thermodynamics when a black hole is present: if we drop ordinary matter into a black hole, it will disappear into a spacetime singularity, together with its entropy $S$. No compensating gain of entropy occurs, so that the total entropy in the universe decreases. One could attempt to salvage the second law by invoking the bookkeeping rule that one must continue to count the entropy of matter dropped into a black hole as still contributing to the total entropy of the universe. However, the second law would then be observationally unverifiable.

Suppose now that the matter that has fallen inside the horizon had quantum correlations with matter that remained outside. How is such a state described by quantum theory? Are these correlations observable? This problem is not yet fully understood, although such correlations play an essential role in giving to Hawking radiation a nearly exact thermal character [1]. It is hard to imagine a mechanism for restoring the correlations during the process of black hole evaporation. On the other hand, if the correlations between the inside and the outside of a black hole are not restored during the evaporation process, then by the time that the black hole has evaporated completely, an initial pure state will have evolved to a mixed state, and some "information" will have been lost.

It has often been asserted that the evolution of an initial pure state into a final mixed state conflicts with quantum mechanics, and this issue is usually referred to as the "black hole information loss paradox." These pessimistic views are groundless. When black hole thermodynamics appeared in the 70's, notions such as POVMs and completely positive maps were unknown to the relativistic community. Today, we know that the evolution of pure states into mixtures is the general rule when a classical intervention is imposed on a quantum system. However, this issue may be conclusively resolved only after there is a consistent theory of quantum gravity, allowing meanwhile for a number of tantalizing speculations. Here we present some of the most popular alternatives of what happens with the "information" when a black hole evaporates.

- Information is lost: this is a fundamental feature of quantum theory in the presence of black holes and not just an effective description. 
- There is no information loss: if the spectrum of Hawking radiation is analyzed carefully, there may be enough non-thermal features to encode all the information. Hod [12] estimated that, under suitable assumptions about black hole quantization, the maximal information emission rate may be sufficient to recover all the information from the resulting discrete spectrum of the radiation.

- Information comes out at the end, at the Plank scale physics. There is a stable black hole remnant with about the Planck mass $(0.02 \mu \mathrm{g})$ and information is somehow encoded in it [13].

Still a different scenario is implied by the works of Gerlach [14] and Boulware [15]: a particle that falls into an eternal black hole crosses the horizon after an infinite amount of the coordinate time $t$, but only a finite amount of its own proper time. On the other hand, the evaporation of a black hole takes a finite amount of the coordinate time, which is the physical time of a distant observer. From the point of view of the infalling observer, the horizon always appears to recede before her, until it finally disappears (or shrinks to the Planck scale) and the region "beyond the horizon" is unattainable. The distant observer sees the infalling one quickly arrive arbitrarily close to the effective horizon, then she is nearly "frozen" there for an exceedingly long time, and finally either the black hole evaporates or the universe collapses.

Acknowledgement. This work was supported by the Gerard Swope Fund.

\section{References}

[1] A. Einstein, B. Podolsky, and N. Rosen, Phys. Rev. 47, 777 (1935).

[2] N. Bohr, Phys. Rev. 48, 696 (1935).

[3] J. S. Bell, Rev. Mod. Phys. 38, 447 (1966).

[4] J. S. Bell, Physics 1, 195 (1964).

[5] D. Bohm, "Quantum Theory" (Prentice-Hall, New York, 1951) p. 614.

[6] C. E. Shannon, Bell System Tech. J., 27, 379, 623 (1948).

[7] C. H. Bennett, Int. J. Theor. Phys. 21, 905 (1982).

[8] R. Landauer, Physics Today 44 (5), 23 (1991).

[9] A. Peres, Phys. Rev. A 61, 022117 (2000).

[10] W. G. Unruh, Phys. Rev. D 14, 870 (1976).

[11] R. M. Wald, Comm. Math. Phys. 45, 9 (1975).

[12] S. Hod, Phys. Lett. A 299, 144 (2002).

[13] Y. Aharonov, A. Casher, and S. Nussinov, Phys. Lett. B 194, 38 (1987).

[14] U. H. Gerlach, Phys. Rev. D 14, 1479 (1976).

[15] D. G. Boulware, Phys. Rev. D 13, 2169 (1976). 